\title{
Performance of a Modified Hybrid Functional in the Simultaneous Description of Stoichiometric and Reduced $\mathrm{TiO}_{2}$ polymorphs
}

\author{
Kyoung Chul Ko, ${ }^{\dagger, \ddagger}$ Oriol Lamiel-García, ${ }^{\dagger}$ Jin Yong Lee, ${ }^{*}, \stackrel{\ddagger}{\ddagger}$ and Francesc Illas $*, \dagger$ \\ ${ }^{\dagger}$ Departament de Química Física \& Institut de Química Teòrica i Computacional (IQTCUB), \\ Universitat de Barcelona, c/ Martí i Franquès 1,08028 Barcelona, Spain. \\ ${ }^{+}$Department of Chemistry, Sungkyunkwan University, Suwon 16419, Korea
}

\begin{abstract}
.-
Conventional density functionals with either the local density approximation (LDA) or generalized gradient approximation (GGA) form of the exchange-correlation potential fail to describe the electronic structure of a large number of metal oxides. Both, LDA and GGA grossly underestimate the band gap of these materials which severely affects the description of oxygen vacancy point defect states in reduced samples. To find a pragmatic approach to simultaneously and accurately describe the atomic and electronic structure of $\mathrm{TiO}_{2}$ most common polymorphs, we explore the effect of the percentage of exact, non-local, Fock exchange on the electronic structure of stoichiometric rutile and anatase. From these results, a modified hybrid functional is proposed to properly describe the atomic structure, formation enthalpy and electronic structure of rutile and anatase and, at the same time, results for reduced samples are also in good agreement with available experimental results. The present approach can be safely used to accurately describe numerous $\mathrm{TiO}_{2}$ based materials containing defects or realistic nanoparticles for which the large unit cells required or system sizes hinder the use of $G W$ related techniques.
\end{abstract}

Keywords. Titanium dioxide, rutile, anatase, oxygen vacancy, DFT, Hartree-Fock exchange, hybrid functional

\section{Corresponding Authors}

*jinylee@skku.edu (Phone: +82-31-299-4560, Fax: +82-31-290-7075), francesc.illas@ub.edu (Phone: +34-93-402-1229. Fax: +34-93-402-1231) 


\section{Introduction}

Titanium dioxide $\left(\mathrm{TiO}_{2}\right)$ has attracted much attention due to its numerous applications such as photocatalysis, ${ }^{1}$ solar cells ${ }^{2}$ and energy and environment. ${ }^{3,4}$ In particular, the discovery by Fujishima and Honda that irradiating $\mathrm{TiO}_{2}$ by light in the ultraviolet region triggers water splitting, ${ }^{5}$ has resulted in enormous efforts in the search for efficient $\mathrm{TiO}_{2}$ based photocatalysts, ${ }^{4}$ and the possibility to prepare photocatalysts with activity under sun light has become a crucial issue. Several recent studies reveal that the presence of point defects in $\mathrm{TiO}_{2}$ greatly influences the electrical $^{6,7}$, optical ${ }^{8,9}$ and photocatalytic properties ${ }^{10-13}$ of this material. Among the different types of point defects in $\mathrm{TiO}_{2}$ and related materials, oxygen vacancies $\left(\mathrm{O}_{\mathrm{v}}\right)$ are known to play a key role in catalytic reactions both as adsorption and active sites. ${ }^{14}$ The $\mathrm{O}_{\mathrm{v}}$ defect is one kind of native defects, and also is easily formed during the preparation of $\mathrm{TiO}_{2}$ materials. ${ }^{15}$ In this regard, the electronic structure of the $\mathrm{O}_{\mathrm{v}}$ defect state in $\mathrm{TiO}_{2}$ systems is intimately connected to their catalytic and photocatalytic performance. Therefore, understanding and controlling the $\mathrm{O}_{\mathrm{v}}$ defect in $\mathrm{TiO}_{2}$ materials could be the fundamental step for designing an efficient photocatalyst.

In spite of the importance of $\mathrm{O}_{\mathrm{v}}$ in $\mathrm{TiO}_{2}$ materials already mentioned, several serious problems exist in the theoretical description of the electronic structure of these systems. In fact, periodic models are needed to properly describe these materials implying the use of methods based on density functional theory (DFT). ${ }^{16}$ However, DFT based methods necessarily make use of an approximate form of the exchange and correlation potential and, because of the common way to compute the Coulomb in the Kohn-Sham implementation of DFT, the calculated results would inherently contain the so-called self-interaction error. ${ }^{17}$ In addition, the conventional local density approximation (LDA) and generalized gradient approximation (GGA) functionals tend to predict highly underestimated band gaps. ${ }^{16}$ This is a general trend usually referred to as the band gap problem in DFT based calculations. This shortcoming of standard LDA and DFT methods results in incorrect description of mid gap states introduced by oxygen vacancies in $\mathrm{TiO}_{2}{ }^{18-20}$ and other reducible oxides. ${ }^{21}$ For $\mathrm{TiO}_{2}$, theoretical studies based on LDA and GGA approaches found that, as a consequence of the severe underestimation of the band gap, ${ }^{18-20}$ the $\mathrm{O}_{\mathrm{v}}$ defect induced energy level was not even located within the band gap region. In addition to the failure in properly describing the position of the defect induced energy levels, LDA and GGA provided a qualitatively incorrect description of the electronic structure of the reduced systems. The creation of $\mathrm{O}_{\mathrm{v}}$ results in the creation of $\mathrm{Ti}^{3+}$ centers which are clearly seen in the most recent electronic 
paramagnetic resonance (EPR) experiments ${ }^{22,23}$ with the assignment confirmed by previous theoretical calculations. ${ }^{24}$ Therefore the extra electrons left on the solid upon oxygen removal are well localized in this $\mathrm{Ti}^{3+}$ centers. The results from LDA and GGA were inconsistent with the experimental observation from diverse spectroscopic techniques based on rutile samples, where the $\mathrm{O}_{\mathrm{v}}$ defect induced energy level appeared $0.7 \sim 1.0 \mathrm{eV}$ below the conduction band minimum $(\mathrm{CBM}),{ }^{25-28}$ and also failed to describe the localized nature of the trapped electrons.

To resolve the problem of abnormal strong delocalization effect on reduced $\mathrm{TiO}_{2}$ systems it is necessary to go beyond the LDA and GGA schemes. In this sense, the DFT $+U$ and a variety of hybrid functionals have been employed to correct, at least in part, the self-interaction error inherent to LDA and GGA in describing the electronic structure of $\mathrm{TiO}_{2}$ systems. ${ }^{29-36} \mathrm{~A}$ comprehensive review has been reported recently by Di Valentin and Pacchioni. ${ }^{37}$ In the DFT $+U$ methods, the U parameter that represents the screened on-site Coulomb interaction of $\operatorname{Ti}(3 \mathrm{~d})$ electrons although a second $\mathrm{U}$ parameter added to $\mathrm{O}(2 \mathrm{p})$ further improves the results. ${ }^{30,38}$ In the hybrid methods, a fraction of non-local Fock exchange is added which, even if applies to all electrons in the system, constitutes an external parameter (for further details see the reviews in Refs. 16 and 37). The amount of Fock exchange to be used constitutes an open issue and it has also been suggested to fix it based on the dielectric constant of the materials. ${ }^{39}$ This approach has been recently used to investigate $\mathrm{TiO}_{2}$ and other semiconducting oxides, ${ }^{40}$ at first sight it may seem a suitable $a b$ initio alternative but one must realize that it also introduces information not included in the first principles Hamiltonian.

Actually, a number of DFT $+U$ and hybrid functional calculations successfully predicted the position of the $\mathrm{O}_{\mathrm{v}}$ energy levels as summarized in the electronic supplementary information (Table S1). In DFT $+U$ method, the results obviously depend on the value of the $U$ parameter which is usually in the $2.0-4.0 \mathrm{eV}$ range. This choice leads to $\mathrm{O}_{\mathrm{v}}$ energy levels between 0.1 and $1.6 \mathrm{eV}$ below the CBM, which seems to be qualitatively correct. However, the calculated band gaps were markedly underestimated over $10 \sim 34 \%$ (see Table S1) and reproducing the experimental band gaps adding just one $U$ term for the Ti(3d) states has not been possible. ${ }^{41}$ In fact, a large $U$ parameter value of $6 \mathrm{eV}$ on $\mathrm{Ti}(3 \mathrm{p})$ and $\mathrm{O}(2 \mathrm{p})$ are required to accurately reproduce the band gap of anatase, rutile and brookite ${ }^{42-44}$ but in this case the states corresponding to $\mathrm{O}_{\mathrm{v}}$ are too deep in the gap and close to the top of the valence band. ${ }^{45}$ On the other hand, hybrid functional normally gives an overestimated band gap for $\mathrm{TiO}_{2}$ polymorphs. In fact, conventional 
hybrid functionals such as the popular B3LYP ${ }^{46}$ containing $20 \%$ of Fock exchange (\%FocK) overestimate the band gap of insulating oxides by $11 \sim 23 \%{ }^{47}$ The PBE0 hybrid functional, ${ }^{48,49}$ adding a $25 \%$ of Fock exchange to the standard PBE form ${ }^{50}$ of the GGA functionals, leads to an even larger overestimation. A more accurate description for the band gap of rutile $(3.05 \mathrm{eV})$ is reached ${ }^{35}$ by means of the so-called screened hybrid functional. This functional, usually referred to as HSE (or HSE06), ${ }^{51,52}$ is a modification of PBE0 and, hence, it contains a $25 \%$ of Fock exchange but introduces a second (screening) parameter. Nevertheless, one must note that subsequent HSE calculations by Déak et al. using the same code and the same supercell report a rather larger value for the band gap of rutile $(3.37 \mathrm{eV}) .{ }^{36}$ The difference between the two HSE calculations can be attributed to the different amount of Fock exchange used; Janotti et al. ${ }^{35}$ used HSE with a reduced value of $20 \%$ of Fock exchange whereas Déak et al. ${ }^{36}$ used the standard $25 \%$ value proposed in the original work of Heyd et al. ${ }^{51,52}$ In any case, different hybrid functionals resulted in the $\mathrm{O}_{\mathrm{v}}$ energy levels between 0.5 and $1.35 \mathrm{eV}$ below CBM (see Table S1). Hence, due to the overestimations of the band gap most hybrid functionals predict $\mathrm{O}_{\mathrm{v}}$ defect state levels too deep in the gap. Many body approaches based on the so-called $G W$ methods have been suggested to overcome the problems exhibited by the DFT methods. However, these methods are computationally expensive and geometry optimization, required to properly describe the structure relaxation induced by the presence of $\mathrm{O}_{\mathrm{v}}$ becomes unfeasible. Consequently, $G W$ calculations are usually carried out on $\mathrm{DFT}+U$ or hybrid optimized structures. ${ }^{53}$

From the discussion above it is clear that $\mathrm{DFT}+U$ and standard hybrid functionals fail to simultaneously reproduce the magnitude of the band gap and position of $\mathrm{O}_{v}$ induced energy levels in $\mathrm{TiO}_{2}$ polymorphs. The difference in the calculated band gap values in the HSE calculations of Janotti et al. ${ }^{35}$ and of Déak et al. ${ }^{36}$ clearly shows that the amount of Fock exchange is a key parameter and may be system dependent. This is in full agreement with previous work showing that a $35 \%$ of Fock exchange is required to properly describe the atomic and magnetic structure of $\mathrm{NiO} .{ }^{54}$ This work strongly suggests tuning the amount of Fock exchange so as to reproduce known observables, as followed by Alkauskas et al. ${ }^{55}$ In this study, we follow this strategy to explore whether a modified hybrid functional is able to simultaneously and correctly describe the crystal and electronic structure of stoichiometric and reduced $\left(\mathrm{O}_{\mathrm{v}}\right.$ containing) $\mathrm{TiO}_{2}$ polymorphs overcoming those drawbacks of DFT method. In order to achieve this goal, firstly we tried to find the optimal value of \%Fock in PBE0 functional to estimate the 
band gaps of bulk rutile and anatase from the Kohn-Sham band structure. One must point out that this is not rigorous since many body effects in the quasi particles are lacking even when using hybrid functionals. However, this is a broadly used approach when $G W$ type calculations are unaffordable. By tuning the \%Fock, Moreira et al. ${ }^{34}$ were able to successfully describe the electronic and magnetic structure of NiO. Very recently, Gerosa et al. ${ }^{40}$ tuned the \%Fock for $\mathrm{TiO}_{2}$ using information from the dielectric constant and found good agreement between the calculated Kohn-Sham gap, $G W$ results and experimental values. In a second step, the newly defined functional containing the optimal \%Fock has been used to calculate the band gap of brookite (not used at the tuning step) and the electronic structures and physical properties for pristine and reduced $\mathrm{TiO}_{2}$ rutile and anatase. The accuracy and benefit of the modified functional of the present approach will be established by comparing the calculated results to those obtained with the standard PBE functional as well as to available experimental data. We expect that the rationally proposed modification of the PBE0 functional will be helpful in the study of photocatalysts based on $\mathrm{TiO}_{2}$ nanoparticles containing hundreds or thousands of atoms.

\section{Materials models and computational details}

Periodic density functional calculations with the standard PBE and with a modified hybrid with various amount of Fock exchange have been carried out for stoichiometric and reduced rutile and anatase polymorphs of $\mathrm{TiO}_{2}$. In the first step, experimental values were used to define the primitive cell parameters of stoichiometric rutile and anatase and single point calculations were carried out to obtain the band gap as a function of the amount of Fock exchange. The unit cell for bulk rutile and anatase contained 6 and 12 atom, respectively. In the second step, geometry optimization for the bulk was carried out using the fraction of Fock exchange which reproduces the band gap of rutile and anatase at the experimental structure. The convergence criteria for geometry relaxations were set to $10^{-3} \mathrm{eV} / \AA$.

The same modified hybrid functional, hereafter denoted as PBEx, was used to study the atomic and electronic structure of reduced rutile and anatase; i.e. supercells containing one oxygen vacancy $\left(\mathrm{O}_{\mathrm{V}}\right)$. To this end, the bulk unit cells were conveniently enlarged to avoid an excessive concentration of oxygen vacancies as described in detail in Section 3.3.

The periodic density functional calculations were carried out including all electrons in the systems with the overall density described by means of numeric atom-centered orbital. For the 
stoichiometric systems, tight grid and tier-2 basis set were employed with scalar-relativistic effects treated at the scaled ZORA level. ${ }^{56}$ Integration in the reciprocal space was carried out using $7 \times 7 \times 7$ and $8 \times 8 \times 8$ gamma centered $k$-point meshes. The same $k$-point meshes were used to compute band structure of each polymorph which, in turn, was used to extract the band gap. For the larger supercells containing $\mathrm{O}_{\mathrm{v}}$ and the concomitant polaronic distortion, we adopted light grid and tier-1 basis set. For the stoichiometric systems, results from this computational setup do not significantly differ from those obtained using more accurate settings, as described in detail in the next section. For the reduced systems, the supercells are large enough so that calculations at $\Gamma$-point converge the total energy to within a tolerance of $10^{-2} \mathrm{eV} / \AA$.

The formation enthalpy $\left(\Delta \mathrm{H}_{\mathrm{f}}\right)$ of pristine $\mathrm{TiO}_{2}$ polymorphs has been computed as

$$
\Delta H_{f}\left(\mathrm{Ti}_{n} \mathrm{O}_{2 \mathrm{n}}\right)=H\left(\mathrm{Ti}_{n} \mathrm{O}_{2 \mathrm{n}}\right)-n H(\mathrm{Ti})-n H\left(\mathrm{O}_{2}\right)
$$

Herein, $H$ denotes the enthalpy for each component. For solids, the contributions for pressure (P) and volume (V) are very small and can be neglected. Thus, we assume that the internal energy of each solid state is almost equivalent to its enthalpy. Equation (1) can be changed as

$$
\Delta H_{f}\left(\mathrm{Ti}_{n} \mathrm{O}_{2 \mathrm{n}}\right)=U\left(\mathrm{Ti}_{n} \mathrm{O}_{2 \mathrm{n}}\right)-n U(\mathrm{Ti})-n H\left(\mathrm{O}_{2}\right)
$$

The value for $U\left(\mathrm{Ti}_{\mathrm{n}} \mathrm{O}_{2 \mathrm{n}}\right)$ is simply used as the calculated energy for pristine $\mathrm{TiO}_{2}$ polymorphs whereas $U(\mathrm{Ti})$ is the calculated atomic energy for hcp bulk Ti which was obtained employing the experimentally obtained crystal structure for Ti solid. ${ }^{57}$ Hence, the lattice parameters were fixed accordingly and atomic positions in the unit cell relaxed with the appropriate functional (PBE or PBEx) with $7 \times 7 \times 7$ gamma centered $k$-point meshes. For the $\mathrm{O}_{2}$ molecule, the enthalpy contribution is obtained as in Eq. (3) based on standard ideal gas, rigid-rotor, and harmonic oscillator approximations.

$$
H(T)-H(0 \mathrm{~K})=\int_{0}^{T} C_{p}\left(T^{\prime}\right) d T^{\prime}=K_{B} T\left(\frac{7}{2}+\frac{\Theta_{v}}{T} \frac{1}{e^{\frac{\Theta}{T}-1}}\right)
$$

where, $\mathrm{T}$ is the absolute temperature and $\Theta_{v}$ is the vibrational temperature. The factor of $7 / 2$ results from ideal gas and rigid-rotor approximation for a homonuclear diatomic molecule. And $\Theta_{v}$ is defined as

$$
\Theta_{v}=\frac{h v}{K_{B}}
$$


where $v$ denotes vibrational frequency, $h$ and $K_{B}$ are Planck's and Boltzmann's constants, respectively. The optimization and vibrational frequency calculations for an oxygen molecule were carried out to obtain this enthalpy contribution using the appropriate functional. Calculations related to formation enthalpy used a more stringent convergence criteria of $10^{-3}$ $\mathrm{eV} / \AA$ for geometrical relaxations. The zero-point energy correction for oxygen also was considered. It should be noted that our calculated enthalpy formation energies are obtained by using the temperature values of standard condition $(T=298.15 \mathrm{~K})$.

All calculations were carried out using the FHI-aims code ${ }^{58,59}$ running on the Marenostrum supercomputer of the Barcelona Supercomputing Center. It is worth pointing out that the calculations with the hybrid functionals (PBE0 or suitable modifications of this functional) are time consuming and required using systematically 512 cores in the case of the primitive cells and 1024 in the largest supercell.

\section{Results and discussion}

\section{1. Defining the PBEx functional}

In a preliminary step, the effect of Fock exchange on the quantitative prediction of the band gap of $\mathrm{TiO}_{2}$ systems was investigated and, in order not to mix different effects, the

experimental crystal structure ${ }^{60,61}$ for rutile and anatase $\mathrm{TiO}_{2}$ bulk polymorphs was employed. Starting with the PBE0 potential having a $25 \%$ of Fock exchange, this parameter was varied from $0 \%$ to $60 \%$ to calculate the band gaps of these materials. We must point out again that the band gap estimated from the Kohn-Sham band structure is not fully justified from theory but it constitutes a useful and pragmatic approach and is the one chosen here.

As seen in Figure 1 and electronic supplementary information (Table S2) the calculated band gap increases with \%Fock. However, the trend follows an apparent linear relationship for both rutile and anatase polymorphs with a slope of 0.098 for both polymorphs. This implies that a $10 \%$ increase in \%Fock increases the calculated PBE band gap by almost $1 \mathrm{eV}$. Hence, the hybrid PBE0 functional, which has $25 \%$ Fock, predicts band gaps of $4.1 \mathrm{eV}$ and $4.4 \mathrm{eV}$ for rutile and anatase, respectively which are substantially larger than the well-known experimental values of $3.03 \mathrm{eV}$ and anatase $3.20 \mathrm{eV}^{62-64}$ although temperature effects cannot be disregarded. ${ }^{65}$ Figure 1 also explains why the band gaps predicted by the PW1PW and B3LYP hybrid functional, 
which contain $20 \%$ of Fock exchange, for $\mathrm{TiO}_{2}$ polymorphs are around $0.5 \mathrm{eV}$ smaller than those by the PBE0 functional (Table S1).

Based on the linear fitting in Figure 1, it is clear that one can tune the \%Fock parameter in the PBE0 functional so as to reproduce the experimental band gap values. It appears that \%Fock values of $12.1 \%$ and $12.9 \%$ reproduce the band gap of rutile and anatase, respectively. These values are sufficiently close to adopt a $12.5 \%$ value for \%Fock exchange, which is an average value for both systems. It should be noted that this value is very close to $13 \%$ of Fock exchange for the hybrid functional, as previously proposed for rutile $\mathrm{TiO}_{2}$ by Zhang et al. ${ }^{66}$ These authors used the exchange and correlation contributions of LDA proposed by Moreira et al. $^{54}$ in their study of electronic and magnetic structure of $\mathrm{NiO}$ and then examined the performance of their modified functional in describing the electronic properties of pristine bulk rutile $\mathrm{TiO}_{2}$ and the stoichiometric and reduced $\mathrm{TiO}_{2}$ (110) surface. The resulting functional with 12.5\%Fock will be referred to as PBEx to distinguish from the standard hybrid PBE0 functional. Note that $12.5 \%$ of Fock exchange corresponds exactly to half the value in the PBE0. To further proof that PBEx is able to properly describe the electronic structure of $\mathrm{TiO}_{2}$ based materials, we have considered the band gap of brookite, another well-known $\mathrm{TiO}_{2}$ polymorph. At the experimental crystal structure ${ }^{67}$ the PBEx calculated band gap is of $3.37 \mathrm{eV}$ which is in good agreement with experimental values in the 3.1-3.4 eV range. ${ }^{68}$ In order to investigate whether PBEx is able to properly describe the main features of $\mathrm{TiO}_{2}$, several properties are computed for stoichiometric and reduced models of rutile and anatase.

\subsection{Validation of PBEx on bulk $\mathrm{TiO}_{2}$ properties}

The amount of Fock exchange defining the PBEx functional has been empirically derived to reproduce the band gap of rutile and anatase at the experimental crystal structure. In order to further validate this approach, the lattice parameters, band gap and formation enthalpies for primitive cells predicted by the hybrid PBEx functional are discussed and compared to experiments and also to those predicted by the standard PBE functional.

The calculated lattice parameters using both PBE and PBEx together to errors with respect to experiment are listed in Table 1. For both rutile and anatase, PBE and PBEx approaches tend to overestimate both $a / b$ and $c$; for PBE case, this overestimation is in line with

previous theoretical results. ${ }^{53,69,70}$ Considering the absolute deviation from the experimental data, 
it can be concluded that PBEx provides highly improved unit cell parameters consistent with experimental values. In addition, the PBEx formation enthalpy $\left(\Delta \mathrm{H}_{\mathrm{f}}\right)$ for rutile and anatase predicted by are closer to experiment than those obtained with the PBE functional; ${ }^{61,62,71,72}$ the maximum errors on the calculated $\Delta \mathrm{H}_{\mathrm{f}}$ significantly decreased from $6.3 \%$ (PBE) to $3.6 \%$ (PBEx) for rutile, and from $4.9 \%$ (PBE) to $2.0 \%$ (PBEx) for anatase, respectively.

The calculated unit cell parameters and physical properties listed in Table 1 validate the reliability of the PBEx functional to describe the geometrical descriptions as well as electronic structures of stoichiometric bulk systems. Though the structural results are similar to those obtained with other hybrid functionals, the electronic structure obtained by PBEx is now comparable to experiment, while both B3LYP and PBE0 significantly overestimate the band gap of both polymorphs. ${ }^{47}$ In the next section we will show that the PBEx functional adequately described reduced $\mathrm{TiO}_{2}$ models as well.

\subsection{PBEx description of the electronic structure of oxygen vacancies in rutile and anatase}

The calculations for the $\mathrm{O}_{\mathrm{V}}$ containing large supercells involve a somehow less stringent basis set, as mentioned above. Results in Table 1 indicate that the effect on the calculated values is sufficiently small. Calculations show that to obtain PBEx converged calculated $\mathrm{O}_{\mathrm{V}}$ formation energies for rutile, supercell sizes larger than $2 \times 2 \times 2$ are required and a $2 \times 2 \times 4$ (95 atoms) supercell has been selected. In a similar way, a $2 \sqrt{2} \times 2 \sqrt{2} \times 1$ (95 atoms) supercell is chosen for anatase. Note that both supercells contain the same number of atoms and, hence, provide the same concentration of $\mathrm{O}_{\mathrm{V}}(1.56 \%)$ which allows for a meaningful comparison. In both cases, the supercells are constructed from the primitive unit cells parameter already optimized for the bulk using either PBE or PBEx functional as reported in Table 1; resulting supercells are displayed in Figure 2 (see also Figures S1 and S2 in the electronic supplementary information).

From a chemical point of view, the removal of one neutral oxygen atom in $\mathrm{TiO}_{2}$ bulk means that three five-fold Ti atoms are formed and two extra electrons are left in the system; these electrons are responsible for the reduction of formally $\mathrm{Ti}^{4+}$ cations to $\mathrm{Ti}^{3+}$. The existence of $\mathrm{Ti}^{3+}$ ions in reduced $\mathrm{TiO}_{2}$ systems were firmly confirmed by many experimental observations, such as color centers, ${ }^{8}$ core level binding energy shifts, ${ }^{73-7475}$ and electron paramagnetic resonance (EPR). ${ }^{76}$ Thus, inducing oxygen vacancy can be one kind of n-type doping of $\mathrm{TiO}_{2}$ samples. From a theoretical point of view, the description of $\mathrm{Ti}^{3+}$ centers in $\mathrm{TiO}_{2}$ systems is not 
straightforward since PBE predicts physically meaningless fully delocalized solutions whereas hybrid functionals predict a variety of near degenerate solutions with a different degree of localization as described in the literature. ${ }^{33}$

At the PBE level, removing of one oxygen atom from rutile resulted in a rather delocalized solution whereas PBEx correctly predicted a solution with two unpaired electrons (coupled high spin) localized in two different $\mathrm{Ti}^{3+}$ centers (Figure 3). For PBEx, substantial spin density is located at symmetry related $\mathrm{Ti}_{4}$ and $\mathrm{Ti}_{4}$, atoms, which are slightly away from $\mathrm{O}_{\mathrm{V}}$ site, instead of $\mathrm{Ti}_{1-3}$ atoms which are the nearest to $\mathrm{O}_{V}$ site. This spin density distribution is in line with recent scanning tunneling microscopy (STM) measurements on the rutile (110) surface reported by Stevin et al., ${ }^{77}$ and with recent theoretical work for reduced rutile using a $2 \times 2 \times 3$ supercell and a similar hybrid functional. ${ }^{78}$ In spite of the different character of the electronic solutions (delocalized versus localized) both PBE and PBEx predict similar optimized geometries for the $\mathrm{O}_{\mathrm{V}}$ containing supercell. In fact, compared to the stoichiometric structure, the atomic distance between $\mathrm{Ti}_{1}$ and $\mathrm{Ti}_{2}$ atoms, hereafter denoted as $l\left(\mathrm{Ti}_{1}-\mathrm{Ti}_{2}\right)$, increases by $0.38 \AA$ for PBE and $0.37 \AA$ for PBEx. In a similar way, $l\left(\mathrm{Ti}_{1}-\mathrm{Ti}_{4}\right)$ and $l\left(\mathrm{Ti}_{2}-\mathrm{Ti}_{4}{ }\right)$ decrease $0.08 \AA$ and $0.06 \AA$ for PBE and PBEx, respectively (Figure 3).

In the case of reduced anatase, the PBE functional predicted the spin density of the two unpaired electrons fully distributed with high spin over all Ti atoms in the supercell (Figure S3). On the contrary, the PBEx hybrid functional predicted almost degenerate spin states with localized unpaired electrons. A similar behavior has been reported by Finazzi et al. using the B3LYP functional and a rather limited basis set of localized GTOs functions. In the present case, three different solutions were found with PBEx. In the first one (I), the spin density arising from the two unpaired electrons is localized over the three $\mathrm{Ti}$ atoms $\left(\mathrm{Ti}_{1-3}\right)$ directly adjacent to $\mathrm{O}_{\mathrm{V}}$ site and is similar to the previously reported solution for antiferromagnetic singlet state obtained by Deák et al. ${ }^{36}$ In the second solution (II), one electron is localized on $\mathrm{Ti}_{1}$ atoms and the remaining spin density is delocalized (II). Finally, a fully delocalized solution similar to that obtained with PBE also found (III). Surprisingly, III is the ground state with II and I being 0.36 and $0.44 \mathrm{eV}$ higher in energy. Solutions (II) and (III) can be obtained starting from I by gradually increasing the bond distance of $l\left(\mathrm{Ti}_{4},-\mathrm{O}_{2}\right)$ to induce symmetry breaking. Unfortunately, all attempts to find a solution with two electrons localized on $\mathrm{Ti}_{1}$ and $\mathrm{Ti}_{4}$, (IV) were failed which was reported as the most stable solution $\left(0.08 \mathrm{eV}\right.$ lower than II) for reduced anatase, as reported by Finazzi et al. ${ }^{33}$. 
In order to analyze the origin of the difference, a single point PBEx calculation was carried out at the optimized structure by Finazzi et al. ${ }^{33}$ that was made available to us by these authors. This PBEx single point calculation converged properly to a solution with two electrons localized (see IV in Figure S3). This result clearly indicates that the PBEx is able to locate the solution with two electrons localized and strongly suggests that the difference comes from a different structure for the supercell with the $\mathrm{O}_{\mathrm{V}}$. In fact, subsequent geometry optimization with PBEx functional starting from this structure led to a slightly different structure where the two electron localization eventually disappeared. It is worthy pointing out that solution IV as reported by by Finazzi et al. $^{33}$ was found for a primitive unit cell with lattice parameters $a, b=3.776 \AA$ and $c=9.866 \AA$ that have much larger deviation from the experimental crystal structure $(a, b=3.785 \AA$ and $c=$ $9.514 \AA$ ) than those predicted by PBEx, especially for the $c$ parameter. Clearly, the existence of IV depends strongly on the use of a given crystal structure and much less on the amount of Fock exchange in the hybrid functional. This result also shows the delicate balance between atomic and electronic structure.

\subsection{Oxygen vacancy formation energy in rutile and anatase}

To further analyze the performance of PBEx functional for reduced $\mathrm{TiO}_{2}$ polymorphs, we calculated the oxygen vacancy formation energy $\left(E_{f}^{O}\right)$ as

$$
E_{f}^{O}=E\left(T i_{n} O_{2 n-1}\right)+\frac{1}{2} E_{O_{2}}-E\left(T i_{n} O_{2 n}\right)
$$

where $E\left(\mathrm{Ti}_{n} \mathrm{O}_{2 n-1}\right)$ and $E\left(\mathrm{Ti}_{n} \mathrm{O}_{2 n}\right)$ represent the total energies of the reduced and pristine $\mathrm{TiO}_{2}$ with fully relaxed supercells, respectively. In the case of the pristine $\mathrm{TiO}_{2}$ both unit cell parameters and fractional coordinates of atoms were fully relaxed. In the case of the $\mathrm{O}_{\mathrm{V}}$ containing unit cell, the lattice parameters for the stoichiometric material were used and the fractional coordinates were fully relaxed. In Eq. (5), $E_{\mathrm{O}_{2}}$ stands for the ground state energy of

oxygen molecule with triplet multiplicity. Herein, a positive value for $E_{f}^{O}$ indicates that the $\mathrm{O}_{\mathrm{v}}$ formation is endothermic reaction. The oxygen vacancy formation energies thus obtained are listed in Table 2. For reduced rutile, the calculated $E_{f}^{O} \mathrm{~s}$ are $4.33 \mathrm{eV}$ and $4.44 \mathrm{eV}$ for PBE and PBEx, respectively. The PBEx calculated value is very close to previously reported values from $\mathrm{GGA}+\mathrm{U}(\mathrm{U}=3 \sim 4 \mathrm{eV})$ approaches, as summarized in Table S1. More importantly, the present 
PBEx calculated $E_{f}^{O}$ value is close to the experimental value of $4.55 \mathrm{eV}$ reported, ${ }^{79}$ which was as an appropriate reference in previous theoretical works. ${ }^{32,33}$

Anatase is more complicated because of the variety of nearly degenerate solutions commented in the previous sections. In the following, we discuss each solution separately but it is important to keep in mind that, for each of them, the atomic structure is different since it corresponds to the fully relaxed geometry. For anatase, the PBE $E_{f}^{O}$ value is $3.69 \mathrm{eV}$ where the PBEx $E_{f}^{O}$ values are in the 4.21 (III) 4.65 (I) $\mathrm{eV}$ range. Here, depending on spin density distribution, the differences in $E_{f}^{O}$ are relatively larger than for rutile. The smaller $E_{f}^{O}$ value predicted from PBE calculations arises from the splitting of spin density in various centers which artificially reduces the coulomb repulsion. Nevertheless, the PBEx values for $E_{f}^{O}$ in anatase are close to those obtained for rutile and are also close to the previously B3LYP reported $E_{f}^{O}$ values of $4.78 \mathrm{eV}$ and 4.84 for anatase (solutions IV and II, respectively). ${ }^{33}$ Clearly, the PBEx functional predicts $E_{f}^{O}$ values intermediate between those obtained from PBE ( $0 \%$ Fock) and B3LYP (20\%Fock) approaches. It is also interesting to note that the range of calculated $E_{f}^{O}$ of PBEx is almost identical to that of $\mathrm{PBE}+\mathrm{U}$ approaches with commonly used $U$ values in the 2-4 eV range (Table $\mathrm{S} 1)$.

From the preceding discussion one can safely affirm that the PBEx calculated $E_{f}^{O}$ values are in the range of values reported in the literature. Note, however, that these values are referred to half the energy of the oxygen molecule. Consequently, a more direct comparison to experiment is difficult.

\subsection{Density of states in oxygen vacancy containing rutile and anatase}

The formation of $\mathrm{O}_{\mathrm{V}}$ is accompanied by a shift of the Fermi level towards the bottom of the conduction band (conduction band minimun or $\mathrm{CBM}$ ) due to the excess electrons. Nevertheless, to properly define the Fermi level a proper alignment with respect to the vacuum is required. ${ }^{64}$ In the present work we simply aligned all calculated DOS by taking the valence band maximum (VBM) as origin. From experiment it is known that, in the case of rutile, the new state, originated by $\mathrm{O}_{\mathrm{V}}$, appears at around $0.7 \sim 1.0 \mathrm{eV}$ below the $\mathrm{CBM}^{25-28}$ although most of the available values correspond to measures for the rutile $\mathrm{TiO}_{2}(110)$ surface. For anatase, resonant 
photoemission and $\mathrm{x}$-ray absorption spectroscopy experiments observed the $\mathrm{O}_{\mathrm{V}}$ defect state around $1.0 \mathrm{eV}$ below the $\mathrm{CBM} .{ }^{80}$

The density of states of reduced titania is one of the key features of these materials since it can be directly compared to experiment. Here, we further analyzed the performance of PBEx functional for reduced $\mathrm{TiO}_{2}$ polymorphs by discussing the calculated total density of state (DOS) for $\mathrm{O}_{\mathrm{v}}$ in $\mathrm{TiO}_{2}$ polymorphs. Let us now discuss the PBE results for the $\mathrm{O}_{\mathrm{v}}$ in the case of rutile and anatase. Figures $4 \mathrm{a}$ and 5a clearly show that PBE predicts the onset of the defect state appears at $0.3 \mathrm{eV}$ below the CBM but merging with the conduction band which is an artifact originated from the excessive delocalization of the spin density by PBE and the underestimation of the band gap. It must be noted that this problem is somehow solved by $\mathrm{DFT}+U$ approaches although the $\mathrm{O}_{\mathrm{v}}$ defect state often appears to deep and close to the valence band maximum; the relevant information is summarized in Table S1. Contrarily to PBE, PBEx properly places the Ov state: $1.15 \mathrm{eV}$ below the CBM for rutile and 0.71 (II) -0.77 (I) $\mathrm{eV}$ below the CBM for the different spin polarized solutions investigated for reduced anatase. Interestingly, I-III solutions for the fully optimized geometry have similar onsets for the $\mathrm{O}_{\mathrm{V}}$ defect state, regardless of degree of localization for the spin density. For the solution IV, as seen in Figure $5 \mathrm{e}$, the $\mathrm{O}_{\mathrm{V}}$ defect state appears $\sim 0.9 \mathrm{eV}$ below the CBM which is consistent with the B3LYP results of Finazzi et al. ${ }^{33}$ and also with the present results for solutions I-III. Therefore, the position of the $\mathrm{O}_{\mathrm{V}}$ related state does not strongly depend on the type of solution and PBEx appears to produce a balanced description for reduced $\mathrm{TiO}_{2}$ as well.

In order to obtain a more accurate estimate of the energy peak in the DOS related to the $\mathrm{O}_{\mathrm{V}}$ defect state, we analyzed the atom-projected and angular-momentum resolved partial density of states (pDOS) focusing especially on $\mathrm{Ti}_{1-4}$ atoms where most of the excess electron density is localized in the $\operatorname{Ti}(3 \mathrm{~d})$ orbitals, especially for PBEx. The maximum of the peak in the pDOS for specific atoms is listed in Table 2. At the PBE level, the $\mathrm{O}_{\mathrm{V}}$ peak maximum appears at $-0.01 \mathrm{eV}$ and $0.1 \mathrm{eV}$ below the CBM for rutile and anatase, respectively. However, from pDOS related to $\mathrm{Ti}_{4}$ and $\mathrm{Ti}_{4}$, atoms, the PBEx predicts the $\mathrm{O}_{\mathrm{V}}$ defect state in rutile $0.89 \mathrm{eV}$ below the CBM in agreement with previous works using $\mathrm{DFT}+U$ or hybrid functionals, ${ }^{34-36,40}$ and with experiment. ${ }^{27,28}$ On the other hand, for anatase, the PBEx calculated $\mathrm{O}_{\mathrm{V}}$ defect states depend on the type of solution found. For solutions I and II involving a rather large degree of localization of the excess electron density, the $\mathrm{O}_{\mathrm{V}}$ peaks appear at 0.49 and $0.41 \mathrm{eV}$ below the CBM, 
respectively. In the case of solution III, exhibiting a large degree of delocalized character, two features at 0.09 and $0.48 \mathrm{eV}$ below the CBM are clearly visible (Figure S4). Finally, in the case of IV, obtained using the unit cell parameters and fractional coordinates from Finazzi et al., ${ }^{33}$ two features at 0.41 and $0.64 \mathrm{eV}$ below the CBM are found. Therefore, in spite of the differences related to different solutions, all physically meaningful results consistently predict the $\mathrm{O}_{\mathrm{V}}$ state in anatase at $\sim 0.5 \mathrm{eV}$ below the $\mathrm{CBM}$ which, as commented below, is consistent with recent findings. ${ }^{78}$ From the comparison above it is clear that the present PBEx approach correctly describes the $\mathrm{O}_{\mathrm{V}}$ state for rutile whereas for anatase the agreement seems not to be as good since the experimental feature appears also at $\sim 1.0 \mathrm{eV}$ below CBM, as in the case of rutile. ${ }^{80}$ At this point it is worth pointing out that a direct comparison to experiment is not straightforward. In fact, Gerosa et al. ${ }^{78}$ argue that this feature may be related to the +1 charged $\mathrm{O}_{\mathrm{V}}$ where upon $\mathrm{O}$ removal only one electron is left on the material.

\section{Conclusions}

Using periodic models and an all electron description with accurate numerical basis sets, a new, property tailored, modified hybrid functional has been proposed as a pragmatic approach to properly describe the atomic and electronic structure of bulk rutile and anatase $\mathrm{TiO}_{2}$ polymorphs and of reduced samples containing one oxygen vacancy. In this approach, the amount of Fock exchange entering into the hybrid functional has been determined based on the existing linear correlation between \%Fock and the (Kohn-Sham) calculated band gap for both rutile and anatase at the experimental structure. Hence, it is found that modifying the PBE0 functional so as to contain a $12.5 \%$ of Fock exchange (PBEx) reproduces the experimental band gaps whereas, at the same Kohn-Sham level, commonly used B3LYP and PBE0 hybrid functionals significantly overestimate them. The resulting PBEx approach also leads to an accurate description of lattice parameters, fractional coordinates for the primitive unit cells as well as for formation enthalpies.

The PBEx functional is also found to properly describe the properties of oxygen vacancy defect in rutile and anatase $\mathrm{TiO}_{2}$ bulk systems. For rutile, the defect state exhibits a physically meaningful localized character and appears $\sim 1.0 \mathrm{eV}$ below the CBM, in agreement with previous work $^{34-36,40}$ and with experiment. ${ }^{27,28}$ For anatase the calculated total density of states (DOS) and atom-projected and angular-momentum resolved partial density of states (pDOS) predict the Ov 
defect state at $\sim 0.5 \mathrm{eV}$ below the CBM. This is in qualitative agreement with experiment although it appears that this value is too close to the CBM; here experiments also point out to an $\mathrm{O}_{\mathrm{V}}$ related feature at $\sim 1.0 \mathrm{eV}$ below the $\mathrm{CBM} .{ }^{80}$ However, it has also been suggested that these feature can be attributed to +1 charged $\mathrm{O}_{\mathrm{V}}$ defects rather than to the neutral $\mathrm{O}_{\mathrm{V}}$ modeled in the present work.

Finally, it is worth pointing out that the amount of Fock exchange empirically determined in this work to be suitable to describe $\mathrm{TiO}_{2}$ systems is in agreement with the values used by Gerosa et al. ${ }^{78}$ based on arguments related to screening of the electron density the dielectric constant of these materials. These authors determine the fraction of Fock exchange self consistently with the static electronic dielectric constant. In such a way, the resulting approach has also an empirical flavor because it makes use of a parameter external to the electronic Hamiltonian. They found that the optimal \%Fock parameters are $15.2 \%$ for rutile and $18.4 \%$ for anatase from dielectic-dependent hybrid method. ${ }^{40}$ The positive part, however, is that the amount of Fock exchange chosen on the basis of the experimental band gap or on the basis of the dielectric constant is very similar and smaller than the values used in standard B3LYP or PBE0. Therefore, the present approach may provide a sufficiently accurate way to study the electronic structure of large $\mathrm{TiO}_{2}$ nanoparticles containing hundreds or thousands of atoms where $G W$ techniques are unaffordable and this is being currently analyzed in our computational laboratory. 


\section{Acknowledgments}

The authors are indebted to Prof. Volker Blum, Dr. Björn Lange, Dr. Sergey Levchenko for their kind advice regarding the calculations with the FHI-aims code and to Dr. Sergio Tosoni for preliminary calculations with the CRYSTAL code regarding the dependence of the band gap with the amount of Fock exchange. The authors wish also to thank Prof. Gianfranco Pacchioni and Dr. Cristiana di Valentin for making their structure of the $\mathrm{O}$ vacancy available to us.

This research was supported by Basic Science Research Program through the National Research Foundation of Korea (NRF) funded by the Ministry of Education (NRF2014R1A6A3A03056449), by the Spanish MINECO grant CTQ2015-64618-R grant and, in part, by Generalitat de Catalunya grants 2014SGR97 and XRQTC and from the NOMAD Center of Excellence project; this project has received funding from the European Union's Horizon 2020 research and innovation programme under grant agreement No 676580. J.Y.L. acknowledges the financial support by Ministry of Science, ICT and Future Planning, subjected project to the project EDISON (Education-research Integration through Simulation On the Net, Grant No. 2012M3C1A6035359). O.L.G is grateful to the Universitat de Barcelona for a predoctoral grant and, FI acknowledges additional support from the 2015 ICREA Academia Award for Excellence in University Research. Computational time at the Marenostrum supercomputer has been provided by the Barcelona Supercomputing Centre through grants from Red Española de Supercomputación and the COMPHOTOCAT project 2014112608 of the Partnership for Advanced Computing in Europe (PRACE).

\section{ASSOCIATED CONTENT}

\section{Electronic Supplementary Information.}

$\dagger$ Electronic supplementary information (ESI) available, Computational details for the formation enthalpy calculations, summary for the calculated band gaps and oxygen vacancy defect states reported in previous literatures, calculated band gaps as a function of the amount of Hartree-Fock exchange, initial/final geometry, calculated energy, spin density distributions, DOS and pDOS for reduced rutile and anatase $\mathrm{TiO}_{2}$ systems. 
Table 1. Experimental (Expt.) and calculated (PBE and PBEx) structural parameters $(a=b, c)$, band gap $\left(\mathrm{E}_{\mathrm{g}}\right)$, and formation enthalpy $\left(\Delta \mathrm{H}_{\mathrm{f}}\right)$ for rutile and anatase using tight grid/tier-2 basis set and light grid/tier-1 basis set levels, respectively; relative deviations with respect to experimental data are given in parenthesis. Distances are in $\AA$ and energies in eV.

\begin{tabular}{|c|c|c|c|c|c|c|c|}
\hline \multirow{2}{*}{$\begin{array}{c}\text { Computational } \\
\text { Level }\end{array}$} & & \multicolumn{3}{|c|}{ Rutile } & \multicolumn{3}{|c|}{ Anatase } \\
\hline & & Expt. & PBE & PBEx & Expt. & PBE & PBEx \\
\hline \multirow{4}{*}{$\begin{array}{l}\text { Tight grid/ } \\
\text { tier-2 basis set }\end{array}$} & $a, b$ & $4.592^{\mathrm{a}}$ & $\begin{array}{c}4.650 \\
(1.3 \%)\end{array}$ & $\begin{array}{c}4.609 \\
(0.4 \%)\end{array}$ & $3.785^{b}$ & $\begin{array}{c}3.799 \\
(0.4 \%)\end{array}$ & $\begin{array}{c}3.792 \\
(0.2 \%)\end{array}$ \\
\hline & $c$ & $2.957^{\mathrm{a}}$ & $\begin{array}{c}2.975 \\
(0.6 \%)\end{array}$ & $\begin{array}{c}2.957 \\
(0.0 \%)\end{array}$ & $9.514^{\mathrm{b}}$ & $\begin{array}{c}9.702 \\
(2.0 \%)\end{array}$ & $\begin{array}{c}9.578 \\
(0.7 \%)\end{array}$ \\
\hline & $\mathrm{E}_{\mathrm{g}}$ & $3.03^{c}$ & $\begin{array}{c}2.07 \\
(31.7 \%)\end{array}$ & $\begin{array}{c}2.97 \\
(2.0 \%)\end{array}$ & $3.20^{\mathrm{d}}$ & $\begin{array}{c}2.14 \\
(33.1 \%)\end{array}$ & $\begin{array}{c}3.22 \\
(0.6 \%)\end{array}$ \\
\hline & $\Delta \mathrm{H}_{\mathrm{f}}$ & $-9.74^{\mathrm{e}}$ & $\begin{array}{c}-9.12 \\
(6.3 \%)\end{array}$ & $\begin{array}{c}-9.39 \\
(3.6 \%)\end{array}$ & $-9.69^{f}$ & $\begin{array}{c}-9.22 \\
(4.9 \%)\end{array}$ & $\begin{array}{c}-9.60 \\
(2.0 \%)\end{array}$ \\
\hline \multirow{2}{*}{$\begin{array}{l}\text { Light grid/ } \\
\text { tier-1basis set }\end{array}$} & $\mathrm{E}_{\mathrm{g}}$ & $3.03^{c}$ & $\begin{array}{c}2.04 \\
(32.7 \%)\end{array}$ & $\begin{array}{c}2.91 \\
(4.0 \%)\end{array}$ & $3.20^{\mathrm{d}}$ & $\begin{array}{c}2.12 \\
(33.8 \%)\end{array}$ & $\begin{array}{c}3.19 \\
(0.3 \%)\end{array}$ \\
\hline & $\Delta \mathrm{H}_{\mathrm{f}}$ & $-9.74^{e}$ & $\begin{array}{c}-9.19 \\
(5.7 \%)\end{array}$ & $\begin{array}{c}-9.47 \\
(2.8 \%)\end{array}$ & $-9.69^{f}$ & $\begin{array}{c}-9.25 \\
(4.5 \%)\end{array}$ & $\begin{array}{c}-9.52 \\
(1.7 \%)\end{array}$ \\
\hline
\end{tabular}

${ }^{\mathrm{a}}$ Ref.62, ${ }^{\mathrm{b}}$ Ref.63, ${ }^{\mathrm{c}}$ Ref. 64, ${ }^{\mathrm{d}}$ Ref. 65, ${ }^{\mathrm{e}}$ Ref. 75 and ${ }^{\mathrm{f}}$ Ref. 76. 
Table 2. Calculated (PBE and PBEx) oxygen vacancy formation energy ( $E_{f}^{O}$ in $\mathrm{eV}$ ) for rutile and anatase and position of corresponding defect states relative to the bottom of the conduction band as obtained from pDOS analysis. In the case of anatase, the different spin polarized solutions analyzed are noted in parenthesis. All energy values are in $\mathrm{eV}$.

\begin{tabular}{|c|c|c|c|c|}
\hline \multirow{2}{*}{} & \multicolumn{2}{|c|}{ Rutile } & \multicolumn{2}{c|}{ Anatase } \\
\cline { 2 - 5 } & $E_{f}^{O}$ & $\mathrm{O}_{\mathrm{v}}$ defect state & $E_{f}^{O}$ & Ov $_{\text {defect state }}$ \\
\hline \multirow{2}{*}{ PBE } & 4.33 & -0.01 & 3.68 & 0.10 \\
& & & $4.65(\mathbf{I})$ & $0.49(\mathbf{I})$ \\
& & & $4.57(\mathbf{I I})$ & $0.41(\mathbf{I I})$ \\
PBEx & 4.44 & 0.89 & $4.21(\mathbf{I I I})$ & $0.09,0.48($ III) \\
& & & & $0.41,0.64(\mathbf{I V})^{\mathrm{a}}$ \\
\hline
\end{tabular}

${ }^{a}$ Single point calculation at the structure reported by Finazzi et al. ${ }^{33}$ 
Figure 1. Calculated band gap $\left(\mathrm{E}_{\mathrm{g}}\right.$ in $\mathrm{eV}$ ) of rutile $(\mathrm{O})$ and anatase $(\bullet) \mathrm{TiO}_{2}$ as a function of \%Fock. A proper linear fit leads to $\mathrm{E}_{\mathrm{g}}=0.098 \times \%$ Fock +1.762 and $\mathrm{Eg}=0.098 \times \%$ Fock + 2.009 for rutile and anatase, respectively and with $\mathrm{r} 2=0.997$ in both cases.

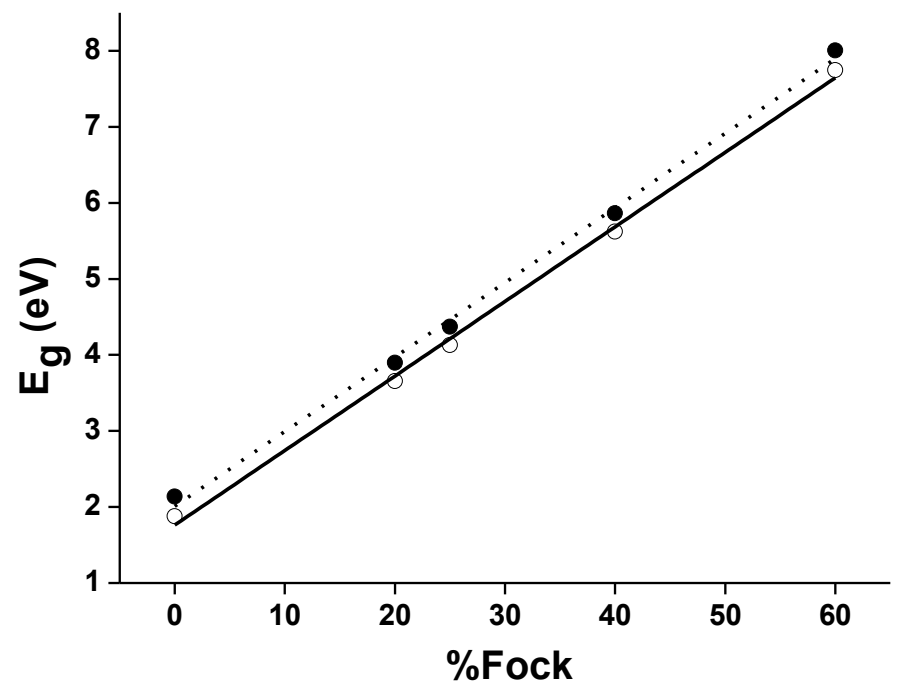


Figure 2. Initial geometries for rutile (a) and anatase (b) containing one oxygen vacancy $\left(\mathrm{O}_{\mathrm{V}}\right.$, denoted by green color). Relevant Ti and $\mathrm{O}$ atoms are labeled.

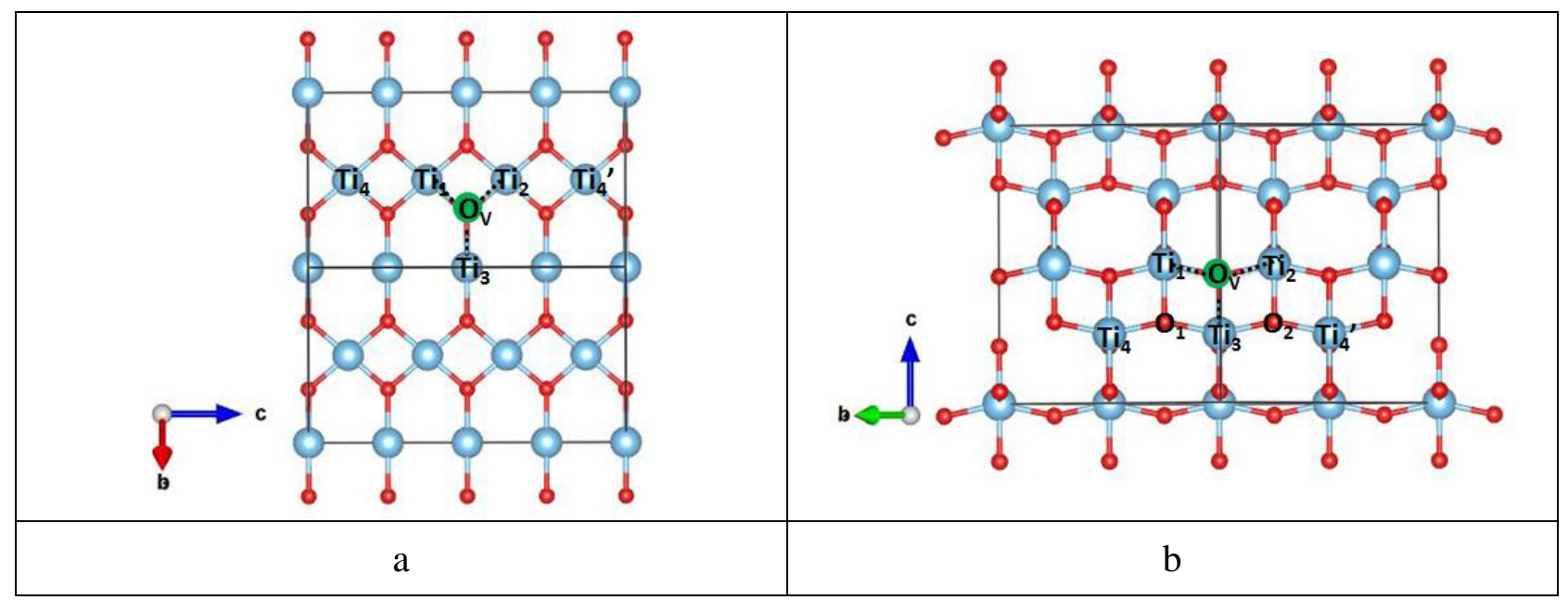


Figure 3. PBE (top panel) and PBEx (bottom panel) optimized geometry and spin density plots for $\mathrm{O}_{\mathrm{V}}$ in rutile. Representative optimized bond lengths (in $\AA$ ) and their changes, before and after one oxygen vacancy involved, noted in parentheses (red color).
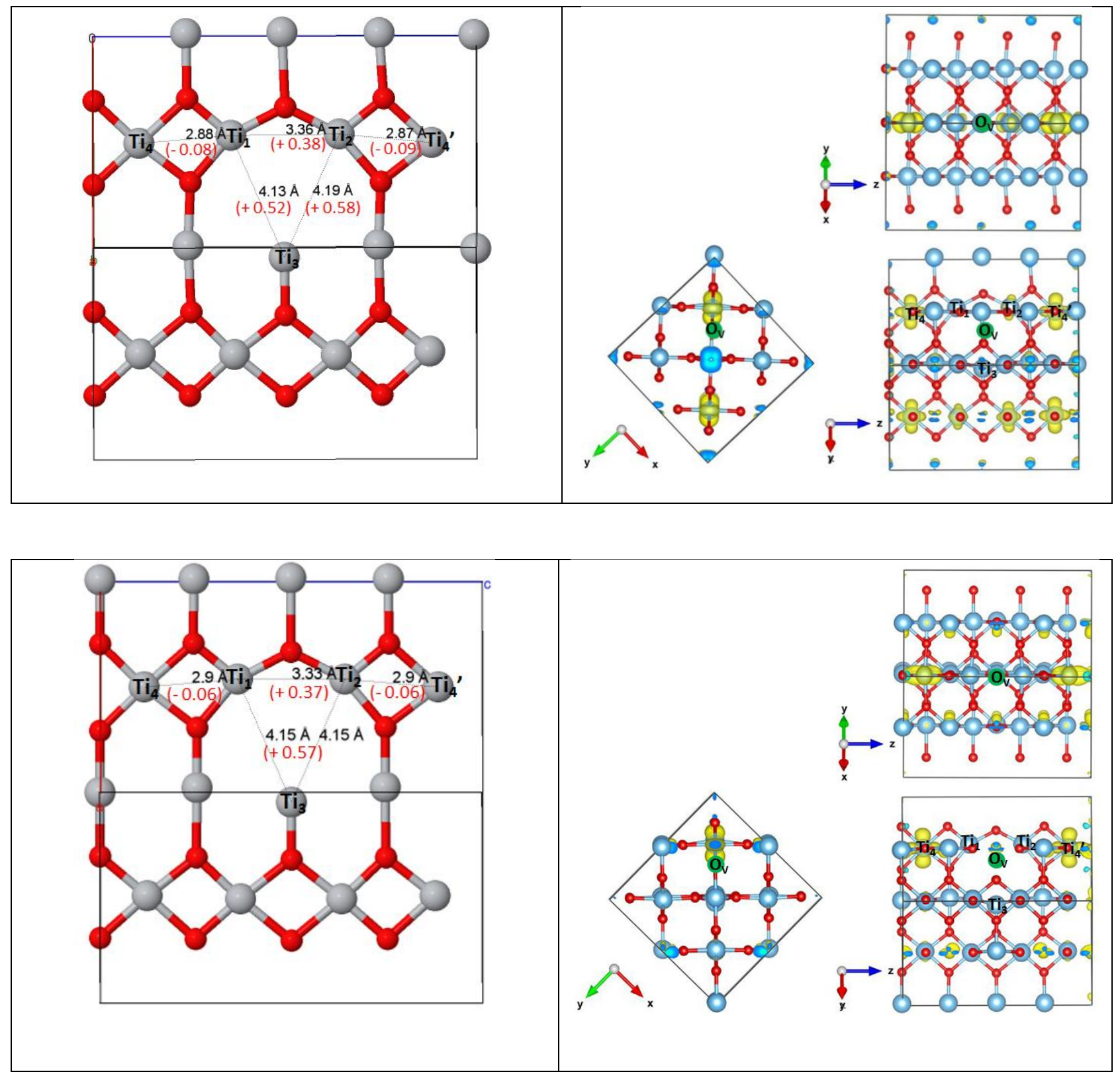
Figure 4. PBE (left) and PBEx (right) total density of states (DOS) for oxygen vacancies $\left(\mathrm{O}_{v}\right)$ in rutile. The blue and red lines represent up-spin and down-spin states, respectively.

anergy (eV)


Figure 5. PBE (a) and PBEx (b-e for solutions I-IV, respectively) total density of states (DOS) for oxygen vacancies $\left(\mathrm{O}_{v}\right)$ in rutile. The blue and red lines represent up-spin and down-spin states, respectively.

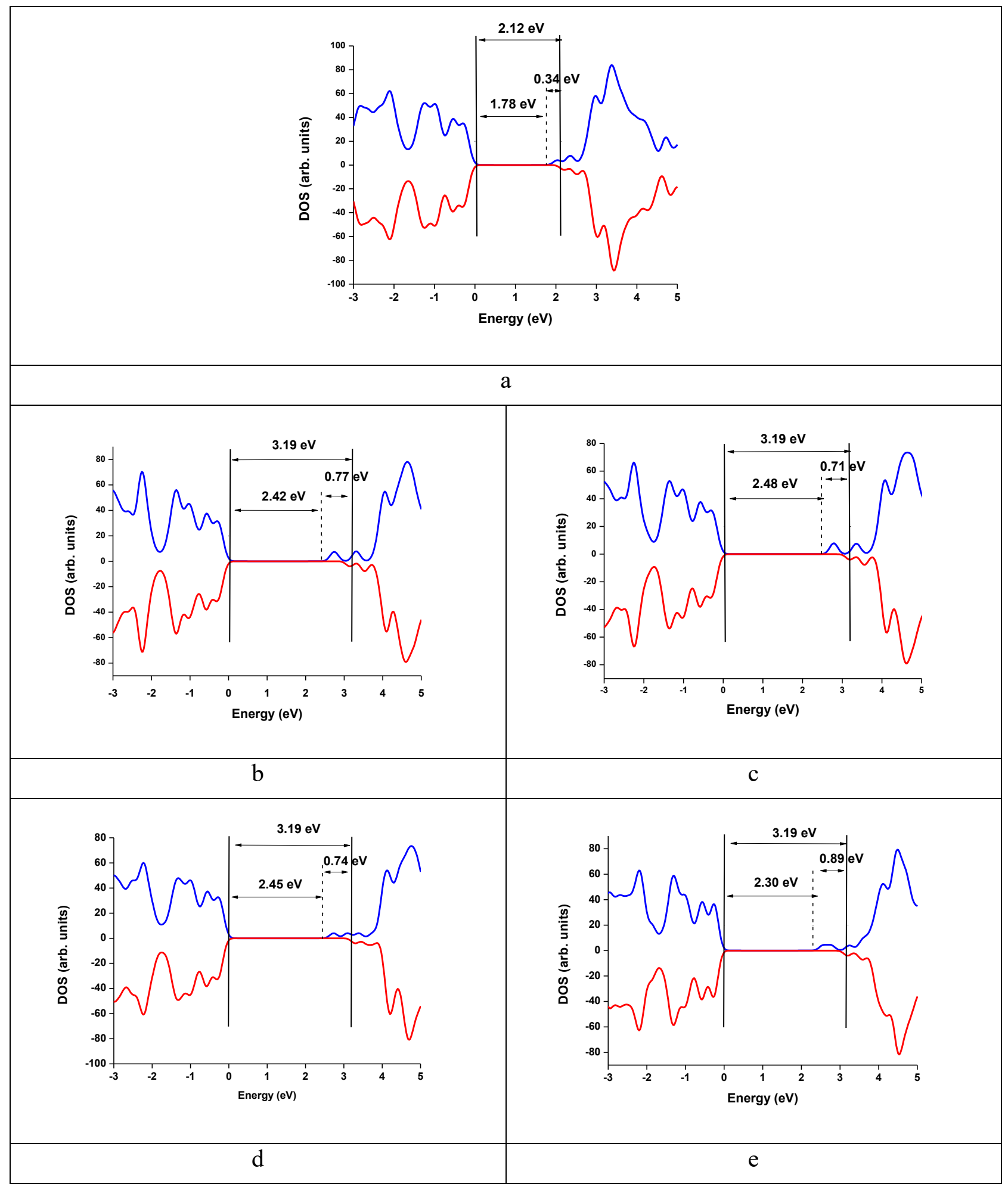




\section{References}

1 A. Mills, N. Elliott, G. Hill, D. Fallis, J. R. Durrant and R. L. Willis, Photochem Photobiol Sci, 2003, 2, 591.

2

B. O'Regan and M. Gratzel, Nature, 1991, 353, 737.

3 T. L. Thompson and J. T. Yates, Jr., Chem. Rev., 2006, 106, 4428.

4 X. Chen and S. S. Mao, Chem. Rev., 2007, 107, 2891.

5 A. Fujishima and K. Honda, Nature, 1972, 238, 37.

6 L. Forro, O. Chauvet, D. Emin, L. Zuppiroli, H. Berger and F. Lévy, J. Appl. Phys. 1994, 75, 633.

7 M. K. Nowotny, T. Bak and J. Nowotny, J. Phys. Chem. B, 2006, 110, 16270.

8 M. V. Khomenko, K. Langer, H. Rager and A. Fett, Phys. Chem. Miner., 25, 338.

9 R. L. Kurtz, S. W. Robey, R. L. Stockbauer, D. Mueller, A. Shih and L. Toth, Phys. Rev. B, 1989, 39, 4768.

10 J. Nowotny, T. Bak, M. Nowotny and L. Sheppard, Int. J. Hydrogen Energy, 2007, 32, 2609.

11 J. Nowotny, T. Bak, M. Nowotny and L. Sheppard, Int. J. Hydrogen Energy, 2007, 32, 2630.

12 C. Di Valentin and G. Pacchioni, Accounts Chem. Res., 2014, 47, 3233.

13 F. Spadavecchia, G. Cappelletti, S. Ardizzone, C. L. Bianchi, S. Cappelli, C. Oliva, P. Scardi, M. Leoni and P. Fermo, Appl. Catal. B-Environ., 2010, 96, 314.

14 S. Polarz, J. Strunk, V. Ischenko, M. W. van den Berg, O. Hinrichsen, M. Muhler and M. Driess, Angew. Chem. Int. Ed. Engl. 2006, 4, 2965

15 X. Pan, M.-Q. Yang, X. Fu, N. Zhang and Y.-J. Xu, Nanoscale, 2013, 5, 3601.

16 C. Sousa, S. Tosoni and F. Illas, Chem. Rev., 2013, 113, 4456.

17 A. J. Cohen, P. Mori-Sanchez and W. Yang, Chem. Rev., 2012, 112, 289.

18 J. M. Sullivan and S. C. Erwin, Phys. Rev. B, 2003, 67, 144415. 
19 E. Cho, S. Han, H.-S. Ahn, K.-R. Lee, S. K. Kim and C. S. Hwang, Phys. Rev. B, 2006, 73, 193202.

${ }^{20}$ S. Na-Phattalung, M. F. Smith, K. Kim, M.-H. Du, S.-H. Wei, S. Zhang and S. Limpijumnong, Phys. Rev. B, 2006, 73, 125205.

21 M. V. Ganduglia-Pirovano, A. Hofmann and J. Sauer, Surf. Sci. Rep., 2007, 62, 219.

22 S. Livraghi, M. Chiesa, M. C. Paganini and E. Giamello, J. Phys. Chem. C, 2011, 115, 25413.

23 M. Chiesa, M. C. Paganini, S. Livraghi and E. Giamello, Phys.Chem. Chem. Phys., 2013, 15, 9435.

24 C. Di Valentin, G. Pacchioni and A. Selloni, J. Phys. Chem. C, 2009, 113, 20543.

25 R. L. Kurtz, R. Stock-Bauer, T. E. Msdey, E. Román and J. De Segovia, Surf. Sci., 1989, 218, 178.

26 V. E. Henrich, G. Dresselhaus and H. J. Zeiger, Phys. Rev. Lett., 1976, 36, 1335.

27 M. Nolan, S. D. Elliott, J. S. Mulley, R. A. Bennett, M. Basham and P. Mulheran, Phys. Rev. B, 2008, 77, 235424.

28 M. A. Henderson, W. S. Epling, C. H. F. Peden and C. L. Perkins, J. Phys. Chem. B, 2003, 107, 534 .

29 G. Mattioli, F. Filippone, P. Alippi and A. Amore Bonapasta, Phys. Rev. B, 2008, 78, 241201.

30 B. J. Morgan and G. W. Watson, J. Phys. Chem. C, 2010, 114, 2321.

31 J. Stausholm-Møller, H. H. Kristoffersen, B. Hinnemann, G. K. H. Madsen and B. Hammer, J. Chem. Phys., 2010, 133, 144708.

${ }^{32}$ G. Mattioli, P. Alippi, F. Filippone, R. Caminiti and A. Amore Bonapasta, J. Phys. Chem. C, 2010, 114, 21694.

33 E. Finazzi, C. Di Valentin, G. Pacchioni and A. Selloni, J. Chem. Phys, 2008, 129, 154113.

34 M. M. Islam, T. Bredow and A. Gerson, Phys. Rev. B, 2007, 76, 045217. 
35 A. Janotti, J. B. Varley, P. Rinke, N. Umezawa, G. Kresse and C. G. Van de Walle, Phys. Rev. $B, 2010,81,085212$.

36 P. Deák, B. Aradi and T. Frauenheim, Phys. Rev. B, 2012, 86, 195206.

37 C. Di Valentin and G. Pacchioni, Acc. Chem. Res., 2014, 47, 3233.

38 Park, S. G.; Magyari-Köpe, B.; Nishi, Y. Phys. Rev. B 2010, 2, 115109.

39 J. H. Skone, M. Govoni and G. Galli, Phys. Rev. B, 2014, 89, 195112.

40 M. Gerosa, C. E. Bottani, L. Caramella, G. Onida, C. Di Valentin and G. Pacchioni, Phys. Rev. B, 2015, 91, 155201.

41 Z. Hu and H. Metiu, J. Phys. Chem. C, 2011, 115, 5841.

42 S. Tosoni, O. Lamiel-Garcia, D. Fernandez Hevia, J. M. Doña and F. Illas, J. Phys. Chem. C, 2012, 116, 12738.

43 S. Tosoni, O. Lamiel-Garcia, D. Fernandez Hevia and F. Illas, J. Phys. Chem. C, 2013, 117, 5855.

${ }^{44}$ Y. Ortega, O. Lamiel-Garcia, D. F. Hevia, S. Tosoni, J. Oviedo, M. A. San-Miguel and F. Illas, Surf. Sci., 2013, 618, 154

45 S. Tosoni, D. Fernandez Hevia, Ó. González Díaz and F. Illas, J. Phys. Chem. Lett., 2012, 3, 2269.

46 A. D. Becke, J. Chem. Phys., 1993, 98, 5648.

47 J. Muscat, A. Wander and N. M. Harrison, Chem. Phys. Lett., 2001, 342, 397.

48 C. Adamo and V. Barone, J. Chem. Phys., 1999, 110, 6158.

49 J. P. Perdew, M. Ernzerhof and K. Burke, J. Chem. Phys., 1996, 105, 9982.

50 J. P. Perdew, K. Burke and M. Ernzerhof, Phys. Rev. Lett., 1996, 77, 3865.

51 J. Heyd, G. E. Scuseria and M. Ernzerhof, J. Chem. Phys., 2003, 118, 8207.

52 A. V. Krukau, O. A. Vydrov, A. F. Izmaylov and G. E. Scuseria, J. Chem. Phys., 2006, 125, 224106. 
53 A. Malashevich, M. Jain and S. G. Louie, Phys. Rev. B, 2014, 89, 075205.

54 I. de P. R. Moreira, F. Illas and R. L. Martin, Phys. Rev. B, 2002, 65, 155102.

55 A. Alkauskas, P. Broqvist, F. Devynck and A. Pasquarello, Phys. Rev. Lett., 2008, 101, 106802.

56 E. van Lenthe, E. J. Baerends and J. G. Snijders, J. Chem. Phys., 1994, 101, 9783.

57 R. W. G. Wyckoff, Crystal structures, Interscience Publishers, New York,, 2d edn., 1963.; p 7-83.

58 V. Havu, V. Blum, P. Havu and M. Scheffler, J. Comput. Phys., 2009, 228, 8367.

59 V. Blum, R. Gehrke, F. Hanke, P. Havu, V. Havu, X. Ren, K. Reuter and M. Scheffler, Comput. Phys. Commun., 2009, 180, 2175.

${ }^{60}$ R. J. Swope, J. R. Smyth and A. C. Larson, Am. Mineral., 1995, 80, 448.

61 C. J. Howard, T. M. Sabine and F. Dickson, Acta Crystallogr. Sect. B, 1991, 47, 462.

62 A. Amtout and R. Leonelli, Phys. Rev. B, 1995, 51, 6842.

63 S. P. Kowalczyk, F. R. McFeely, L. Ley, V. T. Gritsyna and D. A. Shirley, Solid State Commun., 1977, 23, 161.

${ }^{64}$ D. O. Scanlon, C. W. Dunnill, J. Buckeridge, S. A. Shevlin, A. J. Logsdail, S. M. Woodley, C. R. A. Catlow, M. J. Powell, R. G. Palgrave and I. P. Parkin, Nature materials, 2013, 12, 798.

65 H. Tang, F. Levy, H. Berger, and P. E. Schmid, Phys. Rev. B, 1995, 52, 7771.

66 Y.-f. Zhang, W. Lin, Y. Li, K.-n. Ding and J.-q. Li, J. Phys. Chem. B, 2005, 109, 19270.

67 E. Meagher and G. A. Lager, Can. Mineral., 1979, 17, 77.

68 A. Di Paola, M. Bellardita and L. Palmisano, Catalysts, 2013, 3, 36.

69 M. Lazzeri, A. Vittadini and A. Selloni, Phys. Rev. B, 2001, 63, 155409.

${ }^{70}$ F. Labat, P. Baranek, C. Domain, C. Minot and C. Adamo, J. Chem. Phys., 2007, 126, 154703.

71 G. V. Samsonov, The Oxide handbook, IFI/Plenum, New York, 2d edn., 1982. 
${ }^{72}$ M. W. Chase and National Institute of Standards and Technology (U.S.), NIST-JANAF thermochemical tables, American Chemical Society ; American Institute of Physics for the National Institute of Standards and Technology, Washington, DC New York, 4th edn., 1998.

${ }^{73}$ E. Serwicka, M. Schlierkamp and R. Schindler, Zeitschrift für Naturforschung A, 1981, 36, 226.

${ }^{74}$ T. Sekiya, T. Yagisawa, N. Kamiya, D. Das Mulmi, S. Kurita, Y. Murakami and T. Kodaira, J. Phys. Soc. Jpn., 2004, 73, 703.

${ }^{75}$ P. F. Chester, J. Appl. Phys, 1961, 32, 2233.

${ }^{76}$ J. Nerlov, S. V. Christensen, S. Weichel, E. H. Pedersen and P. J. Møller, Surf. Sci., 1997, 371, 321.

${ }^{77}$ M. Setvin, C. Franchini, X. Hao, M. Schmid, A. Janotti, M. Kaltak, C. G. Van de Walle, G. Kresse and U. Diebold, Phys. Rev. Lett., 2014, 113, 086402.

${ }^{78}$ M. Gerosa, C. E. Bottani, L. Caramella, G. Onida, C. Di Valentin and G. Pacchioni, J. Chem. Phys., 2015, 143, 134702.

${ }^{79}$ P. Kofstad, Nonstoichiometry, diffusion, and electrical conductivity in binary metal oxides, Wiley-Interscience, New York,, 1972.; Chap. 8.

${ }^{80}$ A. G. Thomas, W. R. Flavell, A. K. Mallick, A. R. Kumarasinghe, D. Tsoutsou, N. Khan, C. Chatwin, S. Rayner, G. C. Smith, R. L. Stockbauer, S. Warren, T. K. Johal, S. Patel, D. Holland, A. Taleb and F. Wiame, Phys. Rev. B, 2007, 75, 035105. 
Graphic for TOC

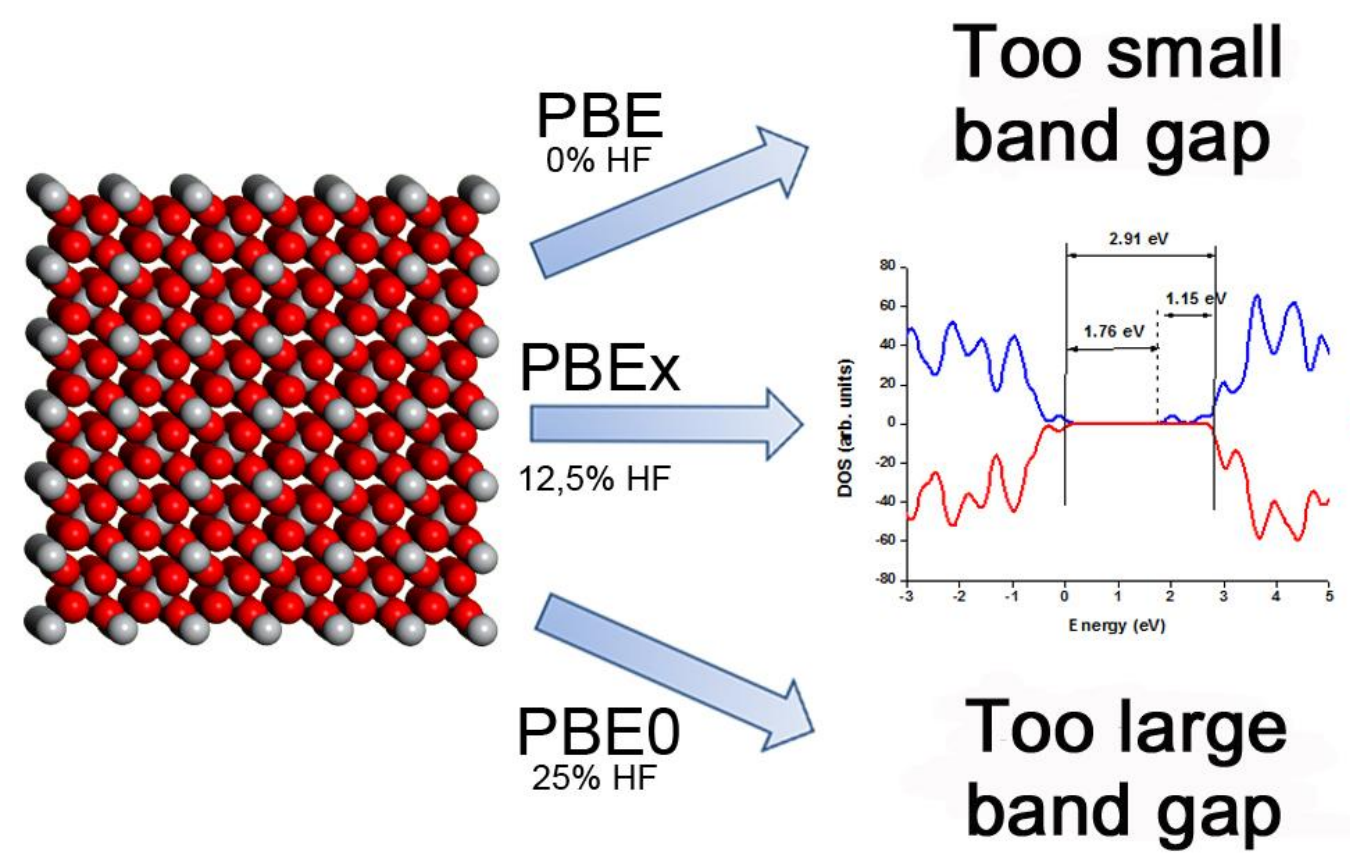

